\title{
Small exponent point groups on elliptic curves
}

\author{
par FLORIAN LUCA, JAMES MCKEE et Igor E. SHPARLINSKI
}

RÉsumÉ. Soit $\mathbf{E}$ une courbe elliptique définie sur $\mathbb{F}_{q}$, le corps fini à $q$ éléments. Nous montrons que pour une constante $\eta>0$ dépendant seulement de $q$, il existe une infinité d'entiers positifs $n$ tels que l'exposant de $\mathbf{E}\left(\mathbb{F}_{q^{n}}\right)$, le groupe des points

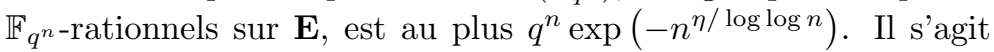
d'un analogue d'un résultat de R. Schoof sur l'exposant du groupe $\mathbf{E}\left(\mathbb{F}_{p}\right)$ des points $\mathbb{F}_{p}$-rationnels, lorsqu'une courbe elliptique fixée $\mathbf{E}$ est définie sur $\mathbb{Q}$ et le nombre premier $p$ tend vers l'infini.

Abstract. Let $\mathbf{E}$ be an elliptic curve defined over $\mathbb{F}_{q}$, the finite field of $q$ elements. We show that for some constant $\eta>0$ depending only on $q$, there are infinitely many positive integers $n$ such that the exponent of $\mathbf{E}\left(\mathbb{F}_{q^{n}}\right)$, the group of $\mathbb{F}_{q^{n}}$-rational points on $\mathbf{E}$, is at most $q^{n} \exp \left(-n^{\eta / \log \log n}\right)$. This is an analogue of a result of R. Schoof on the exponent of the group $\mathbf{E}\left(\mathbb{F}_{p}\right)$ of $\mathbb{F}_{p}$-rational points, when a fixed elliptic curve $\mathbf{E}$ is defined over $\mathbb{Q}$ and the prime $p$ tends to infinity.

\section{Introduction}

Let $\mathbf{E}$ be an elliptic curve defined over $\mathbb{F}_{q}$, the finite field of $q$ elements, where $q$ is a prime power, defined by a Weierstrass equation

$$
Y^{2} Z+a_{1} X Y Z+a_{3} Y Z^{2}=X^{3}+a_{2} X^{2} Z+a_{4} X Z^{2}+a_{6} Z^{3} .
$$

We consider extensions $\mathbb{F}_{q^{n}}$ of $\mathbb{F}_{q}$ and, accordingly, we consider the sets

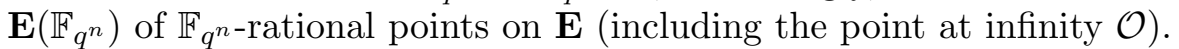

We recall that $\mathbf{E}\left(\mathbb{F}_{q^{n}}\right)$ forms an abelian group (with $\mathcal{O}$ as the identity element). The cardinality $\# \mathbf{E}\left(\mathbb{F}_{q^{n}}\right)$ of this group satisfies the Hasse-Weil inequality

$$
\left|\# \mathbf{E}\left(\mathbb{F}_{q^{n}}\right)-q^{n}-1\right| \leq 2 q^{n / 2}
$$

(see $[2,13,14]$ for this, and other general properties of elliptic curves).

It is well-known that the group of $\mathbb{F}_{q^{n}}$-rational points $\mathbf{E}\left(\mathbb{F}_{q^{n}}\right)$ is of the form

$$
\mathbf{E}\left(\mathbb{F}_{q^{n}}\right) \cong \mathbb{Z} / L \mathbb{Z} \times \mathbb{Z} / M \mathbb{Z}
$$

Manuscrit reçu le 1er janvier 2005. 
where the integers $L$ and $M$ are uniquely determined with $M \mid L$. In particular, $\# \mathbf{E}\left(\mathbb{F}_{q^{n}}\right)=L M$. The number $\ell\left(q^{n}\right)=L$ is called the exponent of $\mathbf{E}\left(\mathbb{F}_{q^{n}}\right)$, and is the largest possible order of points $P \in \mathbf{E}\left(\mathbb{F}_{q^{n}}\right)$.

Trivially, from the definition (1.2), and from the equation (1.1), we see that the inequality

$$
\ell\left(q^{n}\right) \geq\left(\# \mathbf{E}\left(\mathbb{F}_{q^{n}}\right)\right)^{1 / 2} \geq\left(q^{n}+1-2 q^{n / 2}\right)^{1 / 2}=q^{n / 2}-1
$$

holds for all $q$ and $n$.

For a fixed elliptic curve $\mathbf{E}$ which is defined over $\mathbb{Q}$ that admits no complex multiplication, it has been shown by Schoof [11] that the inequality

$$
\ell(p) \geq C(\mathbf{E}) \frac{p^{1 / 2} \log p}{\log \log p}
$$

holds for all prime numbers $p$ of good reduction, where the constant $C(\mathbf{E})>$ 0 depends only on the curve $\mathbf{E}$.

Duke [7], has recently shown, unconditionally for elliptic curves with complex multiplication, and under the Extended Riemann Hypothesis for elliptic curves without complex multiplication, that for any function $f(x)$ that tends to infinity as $x$ tends to infinity, the lower bound $\ell(p) \geq p / f(p)$ holds for almost all primes $p$. However, for elliptic curves without complex multiplication, the only unconditional result available is also in [7], and asserts that the weaker inequality $\ell(p) \geq p^{3 / 4} / \log p$ holds for almost all primes $p$. It has also been shown in [11], that, under the Extended Riemann Hypothesis, for any curve $\mathbf{E}$ over $\mathbb{Q}$,

$$
\liminf _{p \rightarrow \infty} \frac{\ell(p)}{p^{7 / 8} \log p}<\infty
$$

where $p$ runs through prime numbers. This bound rests on an explicit form of the Chebotarev Density Theorem. Accordingly, unconditional results of [9] lead to an unconditional, albeit much weaker, upper bound on $\ell(p)$.

In extension fields of $\mathbb{F}_{q}$, with $\mathbf{E}$ defined over $\mathbb{F}_{q}$, stronger lower bounds on $\ell\left(q^{n}\right)$ can be obtained. For example, it has recently been shown in [10] that for any $\varepsilon>0$, the inequality $\ell\left(q^{n}\right) \leq q^{n(1-\varepsilon)}$ holds only for finitely many values of $n$. In particular, this means that no result of the same strength as (1.3) is possible for elliptic curves in extension fields. Accordingly, here we obtain a much more modest bound which asserts that for some positive constant $\eta>0$ depending only on $q$,

$$
\liminf _{n \rightarrow \infty} \frac{\ell\left(q^{n}\right)}{q^{n} \exp \left(-n^{\eta / \log \log n}\right)}<\infty .
$$

The question of cyclicity, that is, whether $\ell\left(q^{n}\right)=\# \mathbf{E}\left(\mathbb{F}_{q^{n}}\right)$, has also been addressed in the literature. For curves in extension fields, this question has been satisfactorily answered by Vlădut [16]. In the situation where $\mathbf{E}$ is 
defined over $\mathbb{Q}$, the question about the cyclicity of the reduction $\mathbf{E}\left(\mathbb{F}_{p}\right)$ when $p$ runs over the primes appears to be much harder (see $[4,5,6]$ for recent advances and surveys of other related results). In particular, this problem is closely related to the famous Lang-Trotter conjecture.

Finally, one can also study an apparently easier question about the distribution of $\ell(q)$ "on average" over various families of elliptic curves defined over $\mathbb{F}_{q}$ (see $\left.[12,15]\right)$.

Throughout this paper, all the explicit and implied constants in the symbol ' $O$ ' may depend only on $q$. For a positive real number $z>0$, we write $\log z$ for the maximum between 1 and the natural logarithm of $z$.

Acknowledgements. The authors would like to thank Joseph Silverman for useful discussions. During the preparation of this paper, F. L. was supported in part by grants SEP-CONACYT 37259-E and 37260-E, and I. S. was supported in part by ARC grant DP0211459. This paper was written during a visit of I. S. to Royal Holloway, University of London (supported by an EPSRC Visiting Fellowship), whose hospitality is gratefully acknowledged.

\section{The field of definition of torsion points}

Let $\overline{\mathbb{F}}_{q}$ be the algebraic closure of $\mathbb{F}_{q}$. Given an elliptic curve $\mathbf{E}$ over $\mathbb{F}_{q}$, the points $P \in \mathbf{E}\left(\overline{\mathbb{F}}_{q}\right)$ with $k P=\mathcal{O}$ for some fixed integer $k \geq 1$, form a group, which is called the $k$-torsion group and denoted by $\mathbf{E}[k]$. If $\operatorname{gcd}(k, q)=1$, then

$$
\mathbf{E}[k] \cong \mathbb{Z} / k \mathbb{Z} \times \mathbb{Z} / k \mathbb{Z} .
$$

Henceforth, we assume that $\operatorname{gcd}(k, q)=1$, so that (2.1) holds. Let $\mathbb{K}_{k}$ be the field of definition of $\mathbf{E}[k]$, that is the field generated by the coordinates of all the $k$-torsion points, and let $d(k)$ denote the degree of $\mathbb{K}_{k}$ over $\mathbb{F}_{q}$. Then $\mathbb{K}_{k}$ is a Galois extension of $\mathbb{F}_{q}$. Let $\mathcal{G}_{k}$ denote the Galois group of this extension. Having chosen generators $P_{1}, P_{2}$ for the $k$-torsion group, one gets a representation of $\mathcal{G}_{k}$ as a subgroup of $\mathrm{GL}_{2}(\mathbb{Z} / k \mathbb{Z})$ : any element of $\mathcal{G}_{k}$ maps each $P_{i}$ to a $(\mathbb{Z} / k \mathbb{Z})$-linear combination of $P_{1}$ and $P_{2}$ for $i=1,2$.

Although the following statement does not seem to appear in the literature, it is based on an approach which is not new. For example, for the $\mathrm{PGL}_{2}$ analogue, see Proposition VII.2 of [2].

Lemma 2.1. Let $t=q+1-\# \mathbf{E}\left(\mathbb{F}_{q}\right)$. If $r$ is a prime with $\operatorname{gcd}\left(r, q\left(t^{2}-\right.\right.$ $4 q))=1$ and such that $t^{2}-4 q$ is a quadratic residue modulo $r$, then $d(r) \mid(r-1)$.

Proof. Since $r$ does not divide $q, \mathbf{E}[r] \cong \mathbb{F}_{r} \times \mathbb{F}_{r}$, and the above Galois representation exhibits $\mathcal{G}_{r}$ as a subgroup of $\mathrm{GL}_{2}\left(\mathbb{F}_{r}\right)$. Since $\mathbb{F}_{q}$ is a finite 
field, $\mathcal{G}_{r}$ is cyclic, generated by the Frobenius map $\tau(\vartheta)=\vartheta^{q}$. Let $A \in$ $\mathrm{GL}_{2}\left(\mathbb{F}_{r}\right)$ correspond to $\tau$. Now $d(r)$ is the order of $A$ in $\mathrm{GL}_{2}\left(\mathbb{F}_{r}\right)$.

If $A$ is a scalar multiple of the identity matrix, then it has order dividing $r-1$. Otherwise, the characteristic polynomial of $A$ equals its minimal polynomial. Since the relation $\tau^{2}-t \tau+q=0$ holds in the endomorphism ring, we have $A^{2}-t A+q I=0$ over $\mathbb{F}_{r}$, and this must be the minimal polynomial of $A$. Since $t^{2}-4 q$ is a quadratic residue in $\mathbb{F}_{r}, A$ has two distinct eigenvalues in $\mathbb{F}_{r}$, from which the result follows immediately.

We remark that without the condition that $t^{2}-4 q$ is a quadratic residue modulo $r$, similar arguments imply that the relation $d(r) \mid\left(r^{2}-1\right)$ holds for any prime $r$ with $\operatorname{gcd}\left(r, q\left(t^{2}-4 q\right)\right)=1$.

\section{Main result}

Lemma 2.1 immediately implies that $\ell\left(q^{n}\right)=O\left(q^{n} n^{-1}\right)$ infinitely often (namely for each $n=d(r)$, where $r$ is a prime with $\operatorname{gcd}\left(r, q\left(t^{2}-4 q\right)\right)=1$ and such that $t^{2}-4 q$ is a quadratic residue modulo $r$ ). Here, we prove a much stronger bound.

Theorem 3.1. There exists a positive constant $\eta>0$ such that for infinitely many pairs of positive integers $(m, n)$ we have $\mathbf{E}[m] \subseteq \mathbf{E}\left(\mathbb{F}_{q^{n}}\right)$ and

$$
m \geq \exp \left(n^{\eta / \log \log n}\right) .
$$

Proof. We let $\Delta=4\left(t^{2}-4 q\right)$ and we show that there exists a constant $\kappa>0$ such for any sufficiently large $x$ there exists a set of primes $\mathcal{R}$ such that each $r \in \mathcal{R}$ has the properties that

$$
\operatorname{gcd}(r, q)=1 \quad \text { and } \quad r \equiv 1 \quad(\bmod \Delta),
$$

and also that

$$
\# \mathcal{R} \geq \exp (\kappa \log x / \log \log x) \quad \text { and } \quad \operatorname{lcm}\{r-1 \mid r \in \mathcal{R}\} \leq x^{2} .
$$

We follow closely the proof of Proposition 10 of [1]. However, we replace the condition of $r-1$ being squarefree by the conditions (3.1). Namely, let $k_{0}$ be the integer of Proposition 8 of [1]. Assuming that $x$ is sufficiently large, as in Proposition 10 of [1], we define $k_{1}$ as the product of all primes up $0.5 \delta \log x$ for a sufficiently small positive constant $\delta$. We now put $k_{2}=k_{1} / \operatorname{gcd}\left(k_{1}, \Delta\right)$ and finally $k=k_{1} / P\left(\operatorname{gcd}\left(k_{0}, k_{2}\right)\right)$. It is clear that $k_{0} \backslash \Delta k$ (note that we have not imposed the squarefreeness condition, and thus we do not need the condition $k_{0}^{2} \nmid \Delta k$ to hold, as in [1]). For each $d \mid k$, we denote by $A_{d}$ the number pairs $(m, r)$ consisting of a positive integer $m \leq x$ and a prime $r \leq x$, with

$$
\operatorname{gcd}(r, q)=1 \quad \text { and } \quad \operatorname{gcd}(m, k)=k / d,
$$


and which satisfy the system of congruences

$$
m(r-1) \equiv 0 \quad(\bmod k) \quad \text { and } \quad r \equiv 1 \quad(\bmod \operatorname{lcm}(\Delta, d)) .
$$

As in [1], we derive that for some constant $C>0$, the inequality

$$
A_{d} \geq C \frac{x^{2} \varphi(d)}{d k \log x}
$$

holds uniformly in $d$, where $\varphi(d)$ is the Euler function. Repeating the same steps as in the proof of Proposition 10 of [1], we obtain the desired set $\mathcal{R}$ satisfying (3.1) and (3.2). It is clear that $t^{2}-4 q$ is a quadratic residue modulo every $r \in \mathcal{R}$ and thus, by Lemma 2.1, the relation $d(r) \mid(r-1)$ holds for all $r \in \mathcal{R}$.

We now define

$$
m=\prod_{r \in \mathcal{R}} r \quad \text { and } \quad n=\operatorname{lcm}\{r-1 \mid r \in \mathcal{R}\} .
$$

Since, $\mathbf{E}[r] \subseteq \mathbf{E}\left(\mathbb{F}_{q^{n}}\right)$ holds for every $r \in \mathcal{R}$, it follows that $\mathbf{E}[m] \subseteq \mathbf{E}\left(\mathbb{F}_{q^{n}}\right)$. We now derive, from (3.2), that $n \leq x^{2}$, and using the Prime Number Theorem, we get

$$
m \geq \exp ((1+o(1)) \# \mathcal{R}) \geq \exp ((1+o(1)) \exp (\kappa \log x / \log \log x)),
$$

which finishes the proof.

It is now clear that Theorem 3.1 implies relation (1.4).

\section{Applications to Lucas sequences}

Let $u_{n}=\left(\alpha^{n}-\beta^{n}\right) /(\alpha-\beta)$ be a Lucas sequence, where $\alpha$ and $\beta$ are roots of the characteristic polynomial $f(X)=X^{2}+A X+B \in \mathbb{Z}[X]$. Then the arguments of the proof of Theorem 3.1 show that there are many primes $r$ such that $A^{2}-4 B$ is a quadratic residue modulo $r$ and the least common multiple of all the $r-1$ is small. In a quantitive form this implies that, for infinitely many positive integers $n$,

$$
\omega\left(u_{n}\right) \geq n^{\eta / \log \log n}
$$

for some positive constant $\eta>0$, where $\omega(u)$ is the number of distinct prime divisors of an integer $u \geq 2$.

Moreover, given $s \geq 2$ Lucas sequences $u_{i, n}, i=1, \ldots, s$, one can use the same arguments to show that, for infinitely many positive integers $n$,

$$
\omega\left(\operatorname{gcd}\left(u_{1, n}, \ldots, u_{s, n}\right)\right) \geq n^{\eta / \log \log n} .
$$

This generalises and refines a remark made in [3]. In particular, we see that for any integers $a>b>1$, the result of [1] immediately implies that

$$
\operatorname{gcd}\left(a^{n}-1, b^{n}-1\right) \geq \exp \left(n^{\eta / \log \log n}\right)
$$


infinitely often (which shows that the upper bound of [3] is rather tight).

\section{References}

[1] L. M. Adleman, C. Pomerance, R. S. Rumely, On distinguishing prime numbers from composite numbers. Annals Math. 117 (1983), 173-206.

[2] I. Blake, G. Seroussi, N. Smart, Elliptic curves in cryptography. London Math. Soc., Lecture Note Series 265, Cambridge Univ. Press, 1999.

[3] Y. Bugenud, P. Corvaja, U. Zannier, An upper bound for the G.C.D. of $a^{n}-1$ and $b^{n}-1$. Math. Zeitschrift 243 (2003), 79-84.

[4] A. Cojocaru, On the cyclicity of the group of $\mathbb{F}_{p}$-rational points of non-CM elliptic curves. J. Number Theory 96 (2002), 335-350.

[5] A. Cojocaru, Cyclicity of CM elliptic curves modulo p. Trans. Amer. Math. Soc. 355 (2003), 2651-2662.

[6] A. Cojocaru, M. R. Murty, Cyclicity of elliptic curves modulo $p$ and elliptic curve analogues of Linnik's problem. Mathematische Annalen 330 (2004), 601-625.

[7] W. Duke, Almost all reductions of an elliptic curve have a large exponent. Comptes Rendus Mathématiques 337 (2003), 689-692.

[8] P. Erdös, C. Pomerance, E. Schmutz, Carmichael's lambda function. Acta Arith. 58 (1991), 363-385.

[9] J. C. Lagarias, H. L. Montgomery, A. M. Odlyzko, A bound for the least prime ideal in the Chebotarev density theorem. Invent. Math. 54 (1979), 271-296.

[10] F. LuCA, I. E. ShParlinski, On the exponent of the group of points on elliptic curves in extension fields. Intern. Math. Research Notices 23 (2005), 1391-1409.

[11] R. Schoof, The exponents of the group of points on the reduction of an elliptic curve, Arithmetic Algebraic Geometry. Progr. Math. 89, Birkhäuser, Boston, MA, 1991, 325-335.

[12] I. E. ShPARLinski, Orders of points on elliptic curves, Affine Algebraic Geometry. Contemp. Math. 369, Amer. Math. Soc., 2005, 245-252.

[13] J. H. Silverman, The arithmetic of elliptic curves. Springer-Verlag, Berlin, 1995.

[14] J. H. Silverman, J. Tate, Rational points on elliptic curves. Springer-Verlag, Berlin, 1992.

[15] S. G. VhăDUŢ, Cyclicity statistics for elliptic curves over finite fields. Finite Fields and Their Appl. 5 (1999), 13-25.

[16] S. G. VLĂDUT, A note on the cyclicity of elliptic curves over finite field extensions. Finite Fields and Their Appl. 5 (1999), 354-363.

Florian LUCA

Instituto de Matemáticas

Universidad Nacional Autónoma de México

C.P. 58089, Morelia, Michoacán, México

E-mail : fluca@matmor.unam.mx

James McKeE

Department of Mathematics

Royal Holloway, University of London

Egham, Surrey, TW20 0EX, UK

E-mail: james.mckee@rhul.ac.uk

Igor E. SHPARLINSKI

Department of Computing

Macquarie University

Sydney, NSW 2109, Australia

E-mail : igor@ics.mq.edu.au 